\title{
CYCLES DE DÉVELOPPEMENT \\ DE QUELQUES INVERTÉBRÉS BENTHIQUES DANS DES RUISSEAUX DES PYRÊNEES CENTRALES
}

\author{
par P. Lavandier ${ }^{1}$ et J. Dumas ${ }^{1}$.
}

\section{I. - INTRODUCTION}

Les travaux concernant les cycles vitaux des invertébrés d'eau courante font apparaître de nombreuses différences suivant les espèces et leur situation géographique (Hynes 1970). Le facteur température est souvent invoqué pour expliquer ces variations.

Les espèces étudiées dans les Pyrénées appartiennent à plusieurs groupes : Hydracariens (MatTEi 1966), Ephéméroptères (Gurllouzic 1965, Thomas 1970, Thibault 1970), Coléoptères (Berthélemy et de Riols 1965), Trichoptères (DÉcamps 1967), Diptères simuliidae (Neveu 1970). Ces observations concernent des cours d'eau à rythme thermique annuel net.

Les cycles envisagés dans cet article se rapportent à des espèces récoltées dans des milieux à température relativement stable. Trois petits ruisseaux de moyenne altitude $(732 \mathrm{~m})$ dans les Pyrénées centrales ont été étudiés (Lavandier et Dumas 1971). Les caractères de ces ruisseaux peuvent être résumés ainsi :

- Etroits (largeur inféricure à $4 \mathrm{~m})$, courts $(500$ à $1000 \mathrm{~m})$, ils coulent sur les alluvions d'une rivière de montagne : la Neste d'Aure.

- Ils présentent des zones très différentes : vase, végétation diverse à base de cresson et de mousses, graviers.

- Leurs caractéristiques physiques et chimiques sont relativement constantes au cours de l'année : la température varie de $8,5^{\circ} \mathrm{C}$ (février) à $13^{\circ} \mathrm{C}$ (août et septembre), l'amplitude thermique journalière ne dépassant pas $1,5^{\circ} \mathrm{C}$; le débit est constant; la vitesse maximum du courant est inférieure à $30 \mathrm{~cm} / \mathrm{s}$.

1. Laboratoire d'Hydrobiologie, Université Paul-Sabatier, 118, route de Narbonne, 31-Toulouse. 


\section{II. - MATERIEL ET MÉTHODE}

De février 1968 à janvier 1969 neuf séries d'échantillonnages ont été réalisées; à chaque période de relevés, une trentaine de biotopes était explorée; dans chaque type de milieu, le même nombre de prélèvements était effectué sur des surfaces équivalentes de $0,1 \mathrm{~m}^{2}$. Des chasses à adultes (à l'aide de filets et de filet-fauchoirs), à raison d'une par mois en hiver, une tous les quinze jours au printemps et en automne, une hebdomadaire durant les mois d'été ont été réalisées durant la même période.

Le matériel aquatique recueilli est consigné dans la liste suivante, entre parenthèses figure le nombre d'individus capturés.

- Ephéméroptères :

Centroptilum luteolum Müller 1776 (2 302),

Ecdyonurus angelieri Thomas 1968 (387),

Paraleptophlebia submarginata Stephens 1835 (923),

Ephemerella ignita Poda 1761 (1 166).

- Plécoptères :

Protonemura beatensis beatensis Despax 1929 (366),

Amphinemura sulcicollis sulcicollis Stephens 1835 (1 199),

Nemurella picteti Klapalek 1909 (16 040),

Leuctra hippopus Kempny 1889 (516),

Leuctra fusca fusca Linné 1758 (850),

Isoperla sp. (257),

Chloroperla torrentium Pictel 1842 (311).

— Trichoptères :

Rhyacophila denticulata McLachlan 1879 (350),

Plectrocnemia laetabilis McLachlan 1880 (439).

- Coléoptères :

Platambus maculatus Linné 1758 (246).

Les cycles ont été déterminés avec des techniques de mesures différentes selon les groupes envisagés :

- Èphéméroptères : à l'exemple de Guillouzic (1965) nous avons retenu cinq critères et abréviations pour définir et séparer les différents stades larvaires : l, larvules ne présentant pas de fourreaux alaires; $L$, larves dont les fourreaux alaires ne dépassent pas la limite postérieure du thorax; L1 et L2, larves dont les fourreaux alaires atteignent le $1^{\text {er }}$ ou le $2^{\text {e }}$ segment abdominal; $\mathrm{N}$, larves dont les fourreaux alaires dépassent le deuxième segment abdominal ou sont complètement formés. Il s'agit alors de larves-nymphes. 
- Plécoptères : les mesures de la largeur de la capsule céphalique (yeux compris) sont réparties dans des classes de taille de 0,1 en $0,1 \mathrm{~mm}$.

- Trichoptères et Coléoptères : les stades larvaires sont déterminés par mesure de la largeur de la capsule céphalique.

\section{III. - RÉSULTATS}

\section{$1^{\circ}$ Êphéméroptères.}

- Centroptilum luteolum (fig. 1). La population présente constamment des larvules au cours de l'année. L'abondance maximum est relevée en hiver. La période de vol dure 7 mois. Les cinq stades larvaires retenus sont tous présents du mois de mai au mois d'octobre. L'évolution de cette population peut s'interpréter de deux façons :

- la période d'éclosion est très longue avec une croissance rapide de l'espèce durant les beaux jours. Une seule génération se développe par an;

- les dernières pontes donnent des adultes à partir du mois de mai. Les larvules, issues des nouvelles pontes de printemps, se développent rapidement et atteignent le stade imago durant l'été. Il y a alors deux générations dans l'année. Cette deuxième génération serait moins abondante que la première : le nombre de larvules est faible.

- Ecdyonurus angelieri (fig. 2). La période de vol s'étend de mai à novembre. La période d'éclosion est étalée sur plusieurs mois d'octobre à juin. Seuls les prélèvements de juillet, août et septembre ne présentent pas de larves au stade 1. La croissance après l'éclosion est rapide. Les stades $\mathbf{L} 2$ apparaissent brusquement au mois de janvier. Les premières éclosions d'octobrenovembre donncront des nymphes en avril (observées lors de prélèvements de contrôle); les dernières (mai-juin) atteindront le stade adulte en fin de période de vol. Le cycle est annuel.

- Paraleptophlebia submarginata (fig. 3). La période de vol s'étend de mars à novembre avec un maximum d'individus capturés en juin. Les éclosions s'étalent de juillet à mars. Il faut en effet noter que les larvules trouvées en mai paraissent être plus âgées que celles trouvées en mars. L'absence de fourreaux alaires les fait tout de même classer dans la même catégorie. Le développement est rapide durant l'hiver, le stade $L 2$ apparaît dès février, les larves-nymphes dès mars. Le cycle est annuel. 

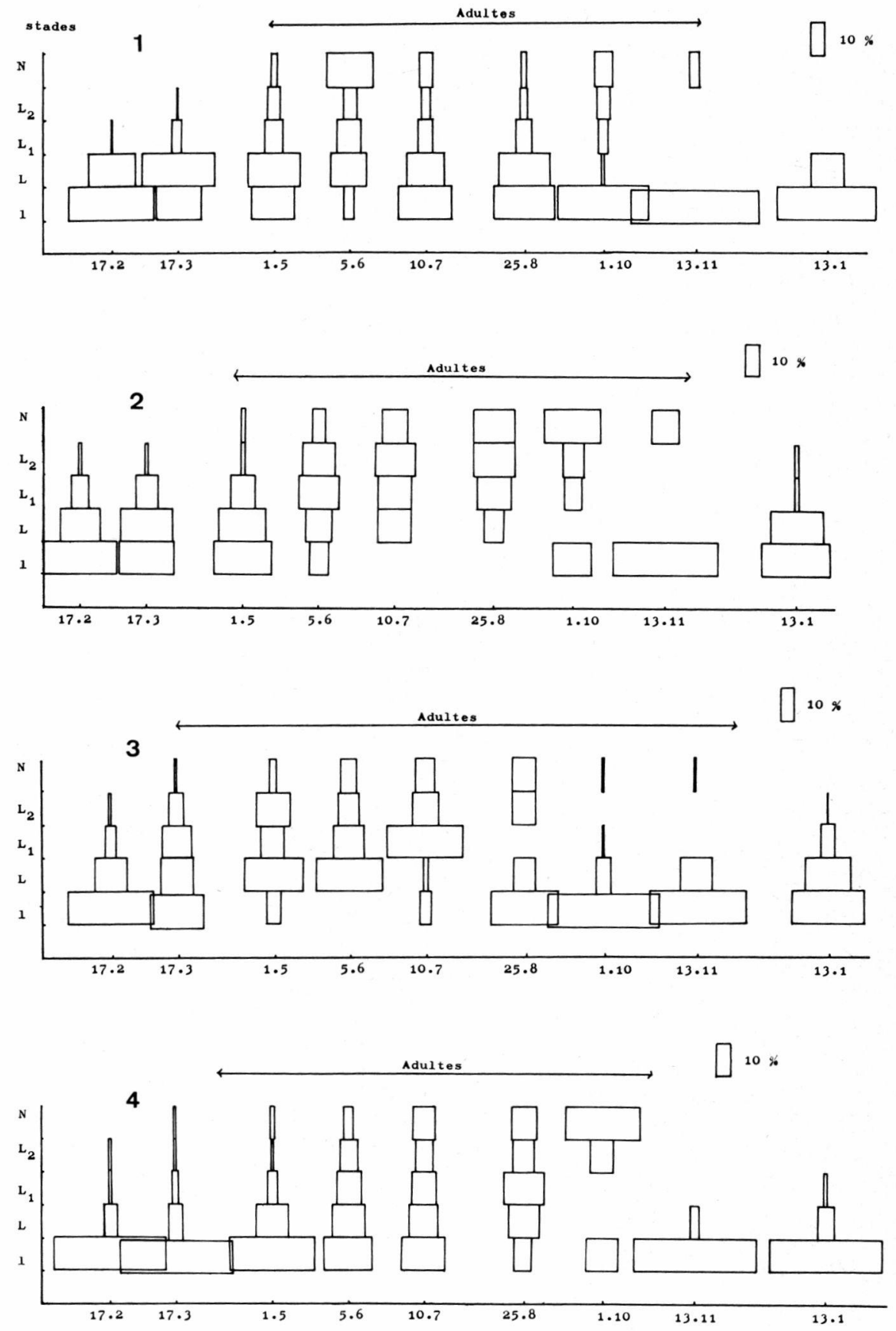

Fig. 1 à 4 . - Cycles de développement. 1: Centroptilum luteolum. - 2 : Ecdyonurus angelieri. - 3: Paraleptophlebia submarginata. - 4: Ephemerella ignita. 
- Ephemerella ignita (fig. 4). La période de vol s'étend d'avril à octobre avec un maximum d'individus en juin. Les larvules sont présentes toute l'année, la majorité d'entre elles a été récoltée aux mois de février, mars, mai. Deux interprétations peuvent être avancées :

- la période d'éclosion est longue, pratiquement étalée sur toute l'annéc. La croissance des larves est régulière. Les premières pontes (avril-mai) donneront des adultes un an plus tard à la même époque. Le cycle est annuel.

- les pontes déposées en été et en automne éclosent et, du fait de la croissance hivernale des larves, les adultes apparaissent au début du printemps. Les nouvelles pontes (mai-juin) éclosent immédiatement et les larves arrivent à l'état adulte en fin de période de vol, une partie pouvant ne pas atteindre la vie imaginale et dégénérer. Deux générations par an sont envisagées dans ce cas.

Ces deux interprétations peuvent être vérifiées simultanément (cf. Thibault 1970).

\section{$2^{\circ}$ Plécoptères.}

- Protonemura beatensis (fig. 5). Il s'agit d'une espèce automnale, quelques adultes ont été capturés en juillet et octobre. Mais des larves âgées apparaissent dès juin et subsistent encore en novembre. Aucune larvule n'a été récoltée du mois de juin au mois de janvier. En juin un large éventail de tailles est représenté. Après l'éclosion, les larvules croissent rapidement. Le cycle biologique annuel présente un arrêt de développement.

- Amphinemura sulcicollis (fig. 6). Les adultes ont été capturés de mai à août, les larvules d'août à novembre. Le maximum d'éclosions a lieu en août. La croissance ralentie en hiver, s'accélère ensuite et les premiers adultes apparaissent début mai. Le cycle est annuel.

- Nemurella picteti (fig. 7). La période de vol est étalée sur 10 mois (février-novembre). La période d'éclosion s'étale sur toute l'année avec toutefois une nette diminution du nombre des larvules en mai et juin. Dans chaque série de prélèvements, la quasi totalité des tailles est représentée. Ces données rendent difficiles l'interprétation du cycle de développement de l'espèce. Il faut noter que la moyenne des mesures des capsules céphaliques des adultes diminue de novembre à août pour les mâles (de $1,16 \mathrm{~mm}$ à $1,10 \mathrm{~mm}$ ), de février à novembre pour les femelles (de $1,43 \mathrm{~mm}$ a $1,22 \mathrm{~mm}$ ). D'autre part, le rapport : nombre de $\hat{s} /$ nombre de $\subsetneq$ effectué sur les adultes capturés est pratiquement constant (entre 

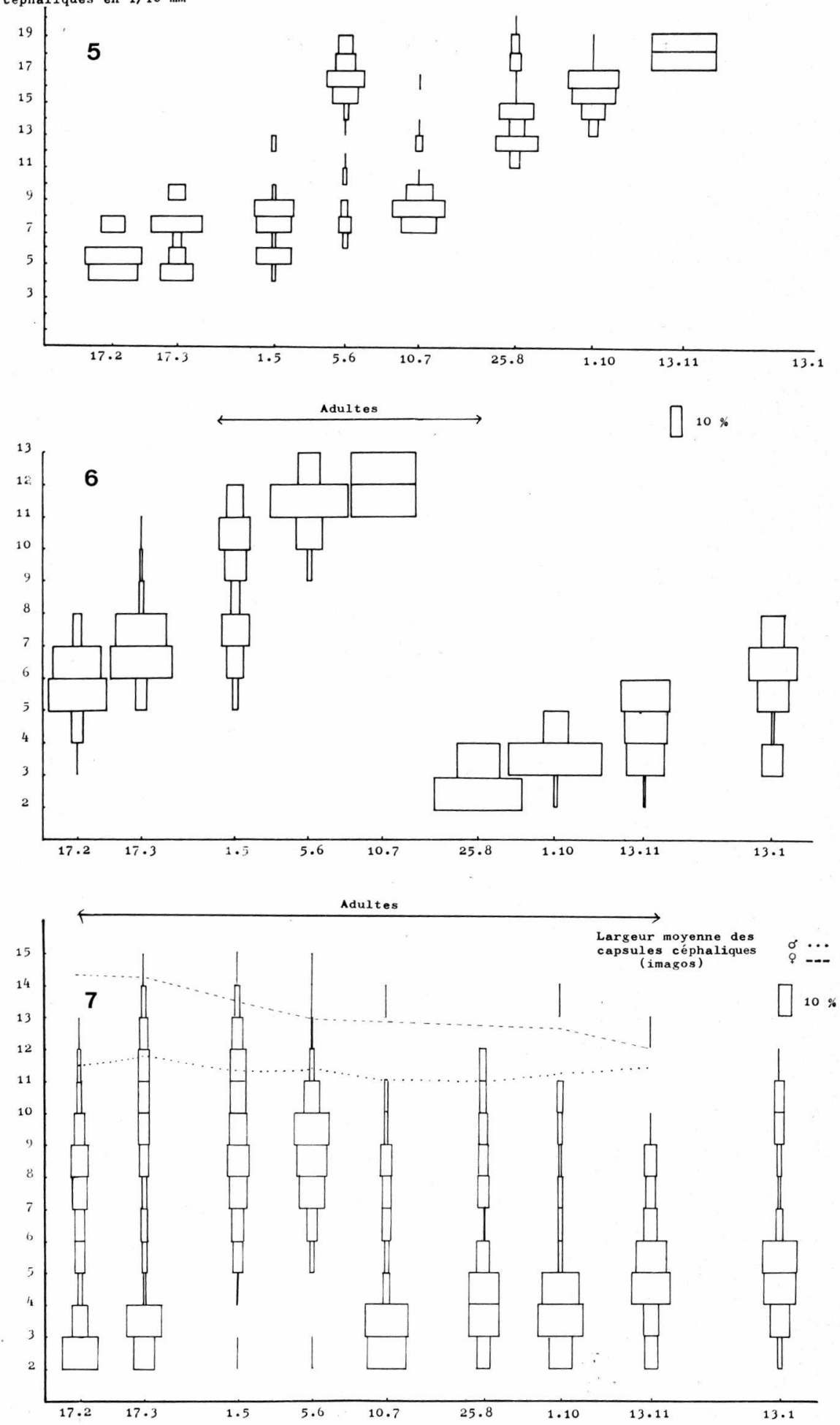

Fig. 5 à 7. - Cycles de développement. 5 : Protonemura beatensis. - 6 : Amphinemura sulcicollis. - 7 : Nemurella picteti. 
1,29 et 1,34). A l'interruption de la période de vol en décembre et janvier semble correspondre une diminution du nombre des larvules en mai-juin. Si l'on admet que les dernières éclosions importantes de mars résultent des dernières pontes de novembre, la durée maximale d'incubation de ces oufs serait de quatre mois et l'espèce pourrait présenter un cycle annuel. Les pontes des derniers adultes éclosent en mars et se développent rapidement, celles des premiers en juillet. La présence de toutes les tailles en juillet-août correspond à un début de développement rapide des larves nouvellement écloses et à la fin du développement des larves qui donneront des adultes en automne.

- Leuctra hippopus (fig. 8.) La période de vol de février à juin fait classer cette espèce parmi les plécoptères printaniers. Les larvules qui apparaissent en juillet croissent de façon régulière. Le cycle est annuel.

- Leuctra fusca (fig. 9). Les éclosions s'étalent de janvier à avril. La croissance des larves, peu importante l'hiver, s'accentue au printemps et en été. Les adultes volent quatre mois (juilletnovembre).

- Isoperla sp. (fig. 10). La période de vol dure cinq mois (mars-juillet). Les plus petites larvules sont trouvées aux mois de juillet, août, octobre et surtout novembre. La croissance ne paraît pas être ralentie durant les mois d'hiver. Le cycle est annuel.

- Chloroperla torrentium (fig. 11). Des adultes ont été capturés de mars à août. Seules quelques larvules dont la largeur de la capsule céphalique était inférieure à $3 / 10 \mathrm{de} \mathrm{mm}$ ont été récoltées. Cependant la présence de larves un peu plus âgées permet d'envisager une durée d'éclosion d'août à mars; le maximum se produisant en automne. La croissance après l'éclosion est rapide. Le cycle de développement est annuel.

\section{$3^{\circ}$ Trichoptères.}

Les deux espèces étudiées présentent 5 stades larvaires (I à V) et un stade nymphal $(\mathrm{N})$ avant de devenir adulte.

- Rhyacophila denticulata (fig. 12). La période de vol est longue, étalée sur huit mois. Des larvules ont été recueillies à chacune des neuf séries d'échantillonnages; leur nombre faible aux mois de mai et juin permet de considérer cette période comme un départ de la nouvelle génération issue des premiers adultes. La croissance est régulière (pour quelques larves, elle pourrait être très rapide à partir dı stade III, quelques-unes parvenant à l'état adulte à l'automne); le stade $\mathrm{V}$ est atteint en novembre. Le 
Largeur des capsules

céphaliques on $1 / 10 \mathrm{~mm}$ Adultes
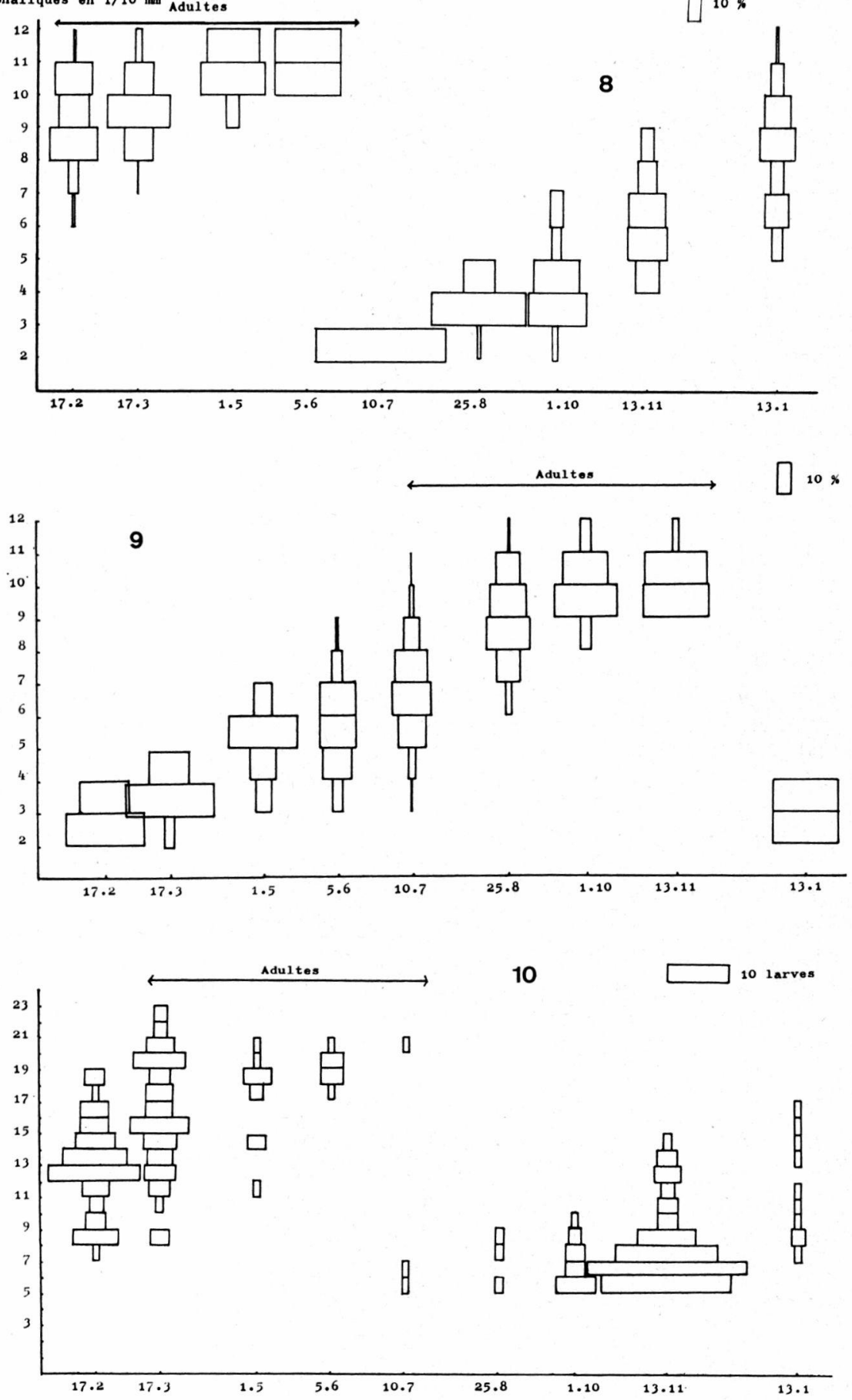

FIG. 8 à 10. - Cycles de développement. 8 : Leuctra hippopus. - 9 : Leuctra fusca. - 10 : Isoperla sp. 
développement semble alors ralenti, les nymphes puis les premiers adultes apparaissent en mars. Le cycle semble annuel, quelques individus pouvant peut-être accomplir tout leur développement durant les mois d'été.

- Plectrocnemia laetabilis (fig. 13). La période de vol s'étend de mai à novembre. Les larvules résultant des premières pontes apparaissent en août et se développent rapidement : le stade $\mathrm{V}$ est atteint en novembre; la transformation en nymphe ne se produit qu'en mars.

\section{$4^{\circ}$ Coléoptères.}

- Platambus maculatus (fig. 14). Les trois stades larvaires sont nettement distincts. La génération qui éclôt d'août à novembre croît rapidement (en janvier le stade III domine et reste seul représenté en mars). Dès le mois de janvier, les individus sont prêts à entrer en nymphose. Les premiers adultes apparaissent en avril-mai, leur nombre augmente jusqu'en juillet, puis décroît pour s'annuler en novembre. Le cycle est annuel.

\section{IV. - DISCUSSION}

Nous avons essayé de classer les cycles de développement observés dans ce milieu d'après les périodes de vol et d'éclosion des diverses espèces. Il faut toutefois noter que si la durée de vie imaginale est relativement aisée à déterminer, la durée de la période d'éclosion ne peut être qu'approchée. La méthode de prélèvement s'est en effet avérée déficiente dans la capture des premiers stades larvaires.

$1^{\circ}$ Cycle des espèces dont la vie larvaire est aquatique, la vie adulte aérienne.

a) Période de vol longue et présence de larvules toute l'année : Centroptilum luteolum, Ephemerella ignita, Rhyacophila denticulata.

II est possible d'envisager une deuxième génération qui, issue des premières pontes de l'année, donnerait des adultes à l'automne. Pour Centroptilum luteolum, BREтsch ко (1965) observe d'ailleurs deux générations annuelles, une d'été à développement rapide, une d'hiver à développement plus lent déterminant deux périodes de vol distinctes en mai-juin et octobre. Ephemerella ignita présente, dans le milieu étudié, un cycle biologique différent de celui décrit généralement : en Grande-Bretagne, Ephemerella ignita 

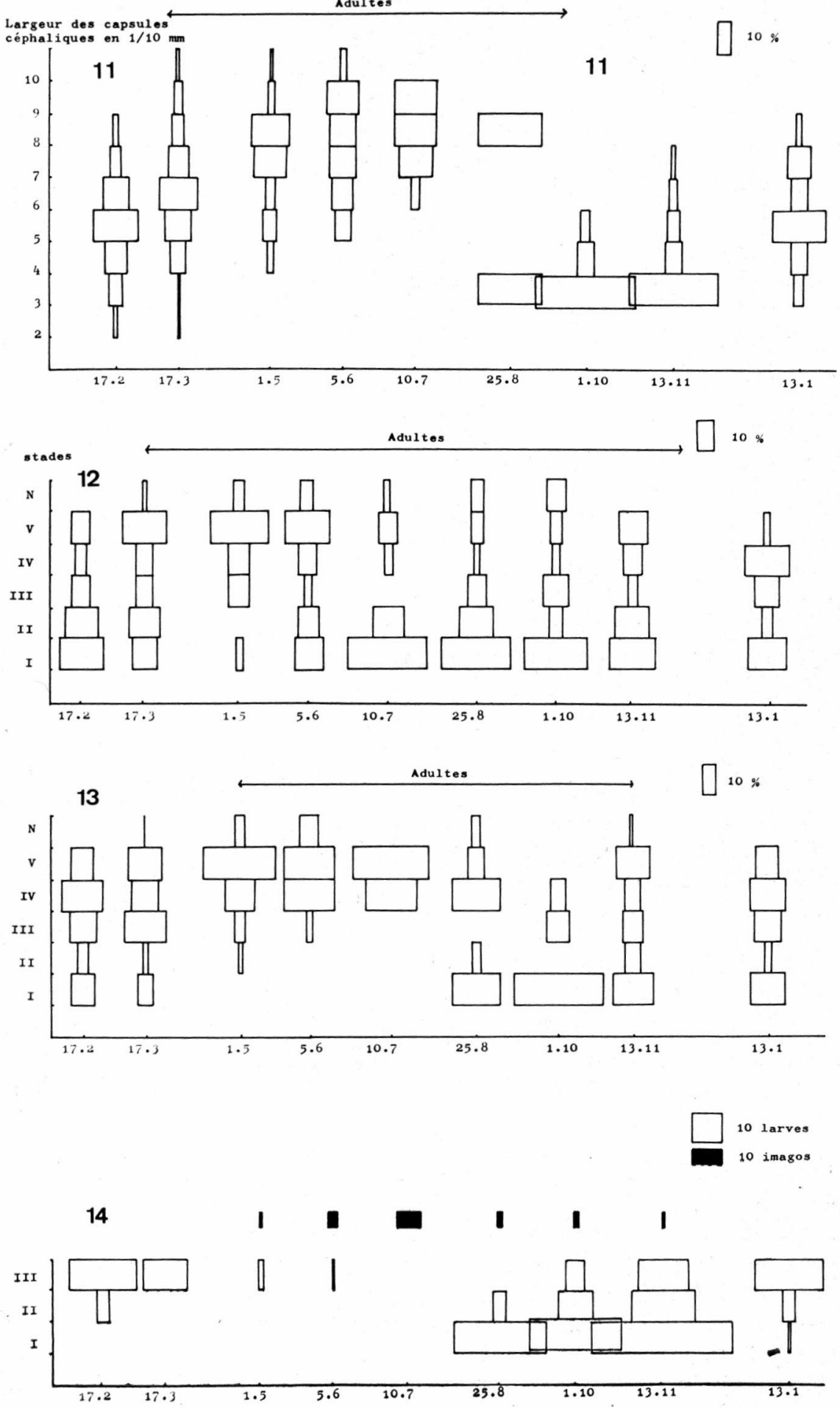

Fig. 11 à 14. - Cycles de développement. 11 : Chloroperla torrentium. 12 : Rhyacophila denticulata. - 13 : Plectrocnemia laetabilis. - 14: Platambus maculatus. 
passe l'hiver au stade œuf, aucune larve n'est observée pendant cette phase de repos de 8 à 9 mois. Le développement est ensuite très rapide : les imagos apparaissent en mars sur l'Afon Hirnant (Hynes, 1961), en mai sur la Wolla Brook (Elliot, 1967), en juin sur une rivière $d u$ «Lake district» (MACAN, 1957) et sur la rivière Endrick (Ecosse), (Maitland, 1965). En Autriche, Pleskot (1958) observe la même évolution : présence de larvules pendant trois mois (mai-juillet), présence d'adultes trois mois (juin-août); quelques larvules assez développées sont recueillies en octobrenovembre. Dans les Pyrénées, Guillovzic. (1965), Thibault (1971) notent la présence de jeunes larves toute l'année et une longue période de vol. Ce dernier auteur, après une étude expérimentale, montre l'importance de la durée de la photopériode sur le développement embryonnaire, une photopériode longue paraissant avoir un effet inhibiteur sur le développement. Il conclut que la plus grande partic de la population d'Ephemerella ignita est univoltine, une faible partie pouvant présenter une seconde génération estivale.

Sur les deux autres espèces de ce groupe (Centroptilum luteolum et Rhyacophila denticulata) une étude expérimentale du temps d'incubation des oufs et de la duréc de développement des larves permettra de préciser le nombre de générations annuelles et leur déterminisme.

b) Périodes de vol et d'éclosion de durée semblable :

- les deux périodes courtes, inférieures à 6 mois : Amphinemura sulcicollis, Leuctra hippopus, Leuctra fusca, Isoperla sp.

Amphinemura sulcicollis : les travaux de Brinck (1949), ElLiot' (1967) rapportent un cycle identique à celui qu'effectue l'espèce à Agos. La période de vol indiquée par BERTHÉLEMY (1966) dans les Pyrénées est plus longue (juin-septembre) mais elle correspond à une période de vol globale pour l'espèce entre 340 et $2160 \mathrm{~m}$ d'altitude. Leuctra hippopus est considérée comme une espèce printanière univoltine. La durée des périodes de vol observées varie dans de faibles proportions : quatre mois (marsjuin) [Brinck 1949]; (février-mai) [Hynes 1961]; cinq mois (février-juin) [BErthéd.evy 1966]; six mois (mars-août) [ELLiotT 1967]. BRINCK et HyNEs notent aussi que la croissance, vigoureuse en automne, décroît quelque peu en hiver. Une croissance printanière puis un fléchissement automnal suivi d'un net ralentissement en hiver sont soulignés par Svensson (1966). Thorup (1963) remarque deux générations annuelles mais envisage, par manque de matériel larvaire, et surtout d'imagos, la confusion entre deux espèces. Leuctra fusca, espèce automnale, présente ụn cycle iden- 
tique à celui enregistré par Brinck (1949) et Hynes (1961) et montre une période de vol décalée d'un mois (en avance) par rapport à celle notée par Bentнélemy (1966).

Comme le remarque ULfstrand (1968) le cycle de ces deux espèces est fondamentalement semblable mais décalé dans le temps, Leuctra fusca a une croissance estivale, Leuctra hippopus une croissance hivernale.

Chez les quatre espèces formant ce groupe nous n'avons pas observé de variations saisonnières de croissance notables. Il semble que le maintien de la température de l'eau proche d'une valeur moyenne $\left(10^{\circ} \mathrm{C}\right)$ détermine un accroissement relativement lent mais régulier et ininterrompu des larves.

- les deux périodes longues, supérieures à 7 mois : Paraleptophlebia submarginata, Nemurella picteti, Plectrocnemia laetabilis.

Paraleptophlebia submarginata: Macan (1957) observe un cycle caractérisé par une période de vol courte (mai-juin) et une période d'éclosion étalée de juillet à février. Les dernières larvules écloses présentent alors une croissance très rapide puisqu'elles atteignent le stade adulte au maximum en quatre mois. Guillouzic (1965) note une période de vol d'avril à octobre et la présence de larvules durant les mois de juillet à novembre. Thibault (1970) envisage un cycle annuel. Les températures hivernales, relativement élevées et supérieures à celles enregistrées en début et fin de saison sur le Volp, expliquent que les adultes de Paraleptophlebia submarginata apparaissent, sur le milieu étudié, un mois plus tôt que sur le Volp, deux mois plus tôt qu'en Angleterre. De plus, les adultes sont présents à une période tardive de l'année (jusqu'en novembre). Brinck (1949) constate que la croissance de Nemurella picteti s'effectue de septembre à mai sans aucun ralentissement hivernal; les adultes volent de mai à septembre. Thorup (1963) met en évidence un fléchissement du développement d'octobre à avril suivi d'une reprise de croissance très rapide puisque les premiers adultes apparaissent en avril; leur vol dure jusqu'en août. La nouvelle génération de larves pourrait apparaître en juillet-août. Dans nos ruisseaux, le cycle de Nemurella picteti est analogue à celui relevé par les différents auteurs. Il apparaît seulement compliqué par une période de vol allongée, due sans doute aux conditions thermiques relativement constantes, qui provoquent un étalement des éclosions. Plectrocnemia laetabilis présente un cycle annuel; sa croissance peut être ralentie en hiver.

Pour ce groupe d'espèces, le développement des premières et des dernières larvules écloses s'effectue dans le même temps. 
c) Période de vol plus courte que la durée d'éclosion : Ecdyonurus angelieri, Chloroperla torrentium.

Chloroperla torrentium présente des durées de vol identiques à celles notées par Hynes (1961) et Elliott (1967) mais des périodes d'éclosion différentes, août-novembre pour Hynes, aoûtavril pour Elliott. Berthélemy (1966) capture des imagos d'avril à octobre. Chloroperla torrentium est un peu plus précoce dans la cressonnière, phénomène sans doute dû à des conditions thermiques de développement plus favorables. Pour cette espèce et Ecdyonurus angelieri, les dernières larvules de printemps semblent boucler leur cycle plus rapidement que celles d'automne.

d) Espèce présentant un arrêt de développement : Protonemura beatensis.

Les œufs pondus en été (ou les jeunes larvules trop petites pour être capturées lors de notre échantillonnage) ne se développeront qu'en février l'année suivante.

$2^{\circ}$ Cycles des espèces dont les larves sont aquatiques, les adultes aquatiques et accessoirement aériens.

Platambus maculatus a une période de reproduction très courte : deux mois et demi au maximum, la vie larvaire s'étale sur une dizaine de mois. Les adultes sont les plus nombreux à la fin du printemps et en été.

\section{V. - CONCLUSION}

HyNEs (1961-1970) a établi une classification des cycles de développement des invertébrés lotiques. Il les caractérise par des lettres $\mathbf{N}$ (cycles non saisonniers), $\mathrm{F}$ (cycles saisonniers rapides), $S$ (cycles saisonniers lents), affectées d'un indice indiquant la période de vie imaginale ou la plus on moins grande complexité apparente du développement.

- Quelques espèces peuvent être aisément replacées dans ces rubriques : Centroptilum luteolum $(\mathrm{Nm})$, Ephemerella ignita $(\mathrm{Nm})$, Rhyacophila denticulata $(\mathrm{Nm})$ présentent de nombreux stades larvaires à chaque période de l'année. Amphinemura sulcicollis $\left(\mathrm{S}_{3}\right)$, Isoperla sp. $\left(\mathrm{S}_{3}\right)$, Leuctra hippopus $\left(\mathrm{S}_{1}\right)$ ont une croissance lente; leurs imagos volent au printemps ou en été.

- Une espèce, Leuctra fusca, a un cycle saisonnier lent avec des adultes fin été, début automne, qui semble justifier l'addition d'un nouvel indice $S_{4}$. 
- Il semble nécessaire pour les autres espèces de préciser, dans le cadre de cette classification, le caractère particulier de leur développement par la juxtaposition de plusieurs lettres ou suffixes numériques. En effet, les amplitudes thermiques faibles, oscillant autour d'une valeur moyenne de $10^{\circ} \mathrm{C}$, déterminent un étalement des périodes d'éclosion et de vie imaginale, et entraînent une croissance en général régulière des individus.

Ainsi, une même espèce correspond à plusieurs des types de cycles de Hynes; elle peut être affectée d'indices différents selon que les premières ou les dernières larves écloses sont considérées.

Par exemple, les premières larvules de Paraleptophlebia, qui donneront les premiers adultes de mars, seront affectées de l'indice $S_{1}$, les dernières donnant les adultes d'octobre-novembre auront l'indice $S_{4}$. Il semble alors possible de caractériser l'espèce par $S_{1}, 2,3,4$.

Les températures relativement stables (particulières aux sources) permettent un développement estival des espèces d'eau froide et déterminent par leurs valeurs hivernales élevées une croissance régulière de la faune à partir d'éclosions largement étalées dans le temps.

\section{RESUMÉ}

- Ie cycle de développement de 14 espèces d'invertébrés benthiques est étudié dans trois petits ruisseaux de moyenne altitude $(732 \mathrm{~m})$ dans les Pyrénées centrales.

- La température reste relativement constante au cours de l'année (minimum $8,5^{\circ} \mathrm{C}$ en février; maximum $13^{\circ} \mathrm{C}$ en août et septembre).

- Différentes classes de tailles ou stades larvaires sont déterminés par observation des fourreaux alaires (Ephémèroptères) ou par mesure de la largeur des capsules céphaliques (Plécoptères, Trichoptères, Coléoptères).

- Les espèces étudiées présentent des périodes d'émergence et d'éclosion en général plus longues ou plus précoces que celles relevées par les divers auteurs; les conditions thermiques entrainent une croissance régulière de la faune.

-- Les cycles, classés d'après la durée des périodes d'éclosion et de vie imaginale sont rapprochés de la classification de Hyses (1961-1970).

Un nouveau type de développement $S_{4}$ correspond à une espèce à croissance lente et période de vol automnale. La juxtaposition de plusieurs indices semble nécessaire pour caractériser le développement larvaire de certaines espèces dont les éclosions sont largement étalées dans le temps. 


\section{LIFE-CYCLES OF BENTHIC INVERTEBRATES IN STREAMS IN THE CENTRAL PYRENEES}

1. The life-cycles of 14 species of benthic invertebrates were studied in three small streams at a mean altitude $(732 \mathrm{~m})$ in the central Pyrenees.

2. The temperature stays fairly constant throughout the year (minimum $8.5^{\circ} \mathrm{C}$ in February; maximum $13^{\circ} \mathrm{C}$ in August and September).

3. The different size classes or larval stages were determined by observing the wing buds (Ephemeroptera) or by measuring the width of the head capsule (Plecoptera, Trichoptera, Coleoptera).

4. The periods of emergence and hatching were generally longer or shorter than those reported by other workers. The temperature conditions caused a regular growth in the fauna.

5. The life-cycles were classified on the basis of the length of the hatching period and adult life, and were compared by the classification of Hynes (1961-1970).

A new type of development $\left(S_{4}\right)$ was found for a species with slow growth and an autumnal flight period. The juxtaposition of several factors seemed necessary to distinguish the larval development of certain species in which hatching continued for a long time.

\section{ENTWICKLUNGSZYKLEN EINIGER BENTHISCHER WIRBELLOSER AUS BÄCHEN DER ZENTRALPYRENÄEN}

1. Es wurde der Entwicklungszyiklus von 14 Wirbellosen dreier kleiner Bäche aus mittlerer Höhe $(732 \mathrm{~m})$ in den Zentralpyrenäen untersucht.

2. Die Temperatur bleibt im Laufe des Jahres ziemlich konstant.

3. Die Einteilung nach Größenklassen oder Larvalstadien erfolgte bei Eintagsfliegen aufgrund der Flügelscheiden, bei Steinfliegen, Köcherfliegen und Käfern durch Messung der Kopfikapsel.

4. Die Schlupfperioden der untersuchten Arten sind entweder länger oder die Schlupftermine früher als in der Literatur angegeben. Die thermischen Bedingungen bewirken ein gleichmäBiges Wachstum.

5. Die Zyklen, nach Schlupfdauer und Dauer der Imaginalzeit klassifiziert, kommen der Klassifikation von Hynes (1961-1970) nahe.

Ein neuer Entwicklungstyp, $S_{4}$, entspricht einer Art langsamen Wachstums und herbstlicher Flugzeit. Die gleichzeitige Verwendung mehrerer Indizes erscheint für die Charakterisierung der Larvalentwicklung gewisser Arten angezeigt, deren Schlupfzeit sich lang hinzieht. 


\section{TRAVAOX CITÉS}

BeRTHélemY (C.). 1966. - Recherches écologiques et biogéographiques sur les Plécoptères et Coléoptères d'eau courante (Hydraena et Elminthidae) des Pyrénées. Annls Limnol., 2 (2) : 227-458.

Berthélemy (C.) et de Riols (J.). 1965. - Les larves d'Elmis du groupe d'E. maugetii (Coléoptères Dryopoidae). Annls Limnol., 1 (1) : 21-38.

Bretsch ko (G.). 1965. - Zur Larvenentuwicklung von cloeon dipterum cloeon simile, Centroptilum luteolum, Baetis rhodani. Z. Zool., 172.

BrinclK (P.). 1949. - Studies on swedish stoneflies (Plecoptera). Opusc. ent., suppl. $11: 250 \mathrm{p}$.

Décamps (H.). 1967. - Ecologie des Trichoptères de la vallée d'Aure (Hautes-pyrénées). Annls Limnol., 3 (3) : 399-577.

ElliotT (J. M.). 1967. - The life histories and drifting of the Plecoptera and Ephemeroptera in a Dartmoor stream. J. Anim. Ecol., 36 : 343-362.

Guit.louzic (M.). 1965. - Cycles de développement de quelques Ephéméroptères du Volp. D.E.S. Fac. Sc. Univ. Toulouse : 34 p.

HyNES (H.B.N.). 1961. - The invertebrate fauna of a Welsh mountain stream. Arch. Hydrobiol., 57 : 344-388.

Hynes (H. B. N.). 1970. - The ecology of running waters. Liverpool university press.

Lavandier (P.) et Dumas (J.). 1971. - Microrépartition de quelques espèces d'invertébrés benthiques dans des ruisseaux des Pyrénées centrales. Annls Limnol., 7 (1) : 7-23.

Macan (T. T.). 1957. - The life histories and migrations of the Ephemeroptera in a stony stream. Trans. Soc. Br. Ent., 12 : 129-156.

Macan (T. T.). 1961. - A key to the Nymphs of the British species of Ephemeroptera. Freshw. Biol. Ass. Scient. Publ., 20 : 1-64.

Maitland (F.S.). 1965. - The distribution, life cycle and predators of Ephemerella ignita (Poda) in the river Endrick-Scotland. Oikos, $16:$ 48-57.

Mattei (G.). 1966. - Les Hydracariens du Massif du Néouvielle. D.E.S. Fac. Sc. Univ. Toulouse : 36 p.

Neveu (A.). 1970. - Ecologie des Simuliidae (Diptera, Nematocera) d'un ruisseau des Pyrénées atlantiques : le Lissuraga. Thèse $3^{\mathbf{e}}$ cycle Fac. Sc. Paris.

Pleskor (G.). 1958. - Die Periodizität einiger Ephemeropteren der Schwechat. Wass. Abwass. : 1-32.

Svensson (P.-O.). 1966. Growth of nymphs of stream living stoneflies in northern sweden. Oikos, $17: 197-206$.

Thibault (M.). 1971. - Le développement des Ephémèroptères d'un ruisseau à truites des Pyrénées atlantiques, le Lissuraga. Annls Limnol., 7 (1) : 53-120.

Thomas (A.). 1970. - Taxonomie et répartition des Ephémèroptères et de quelques Diptères aquatiques (Tipuloidea et Psychodidae) des Pyrénées. Thèse $3^{\mathrm{c}}$ cycle, Fac. Sc. Toulouse.

Thorup (J.). 1963. - Growth and life-cycle of invertebrates from Danish springs. Hydrobiologia, $22(1 / 2)$ : 55-84.

Ulfstrand (S.). 1968. - Life cycles of benthic insects in Lapland streams (Ephemeroptera, Plecoptera, Trichoptera, Diptera : Simuliidae). Oikos, $19: 167-190$. 